DEMOGRAFIA DE DOS JARAS MEDITERRANEAS (Cistus albidus L, Y Cistus clusii DUNAL) EN EL Rosma rino-Ericion DE LA SIERRA DE CREVILLENTE (PROVINCIA DE ALICANTE)

Por J.BELLOT' ${ }^{1}$ y A.ESCARRE ${ }^{1}$

\title{
INTRODUCCION
}

E1 presente trabajo forma parte de un estudio más amplio que se lleva a cabo en el Departamento de Bio logía de la Facultad de Ciencias de la Universidad de $\bar{A}$ licante, sobre el matorral mediterráneo, y que abarca as pectos de la biomasa, producción, reproducción, demogra fía y ocupación del espacio, de algunas de las especies cuantitativamente más importantes.

En enfoque demográfico, muy raramente utilizado por los botánicos con anterioridad a los años setenta, ha sido sin embargo objeto principal de estudio de varios autores durante la pasada década . Entre las prime ras publicaciones están lás de Sarukhan y Harper (1973), Sarukhan y Gadgil (1974), que centran el problema en los diferentes modos de reproducción y en el flujo de la po blación; es sin duda Harper (1977) el que centra defin $\bar{i}$ tivamente el estudio de la demografía vegetal, aportando datos y modelos de estudio para un amplio espectro de es pecies . Posteriormente, los trabajos de Werner y Caswell (1977), Watkinson y Harper (1978), Caswell y Werner(1978) Hubbell y Werner (1979), plantean la demografía vegetal desde el punto de vista de la autorregulación de las po blaciones y de la capacidad de predicción que se tiene a partir de estudios instantáneos .

1. Departamento de Biología . Facultad de Ciencias . Univ. Alicante 
Es muy probable que el dinamismo que comporta el enfoque demográfico en los vegetales, tradicionalmente vistos por los ecólogos como organismos excesivamente es táticos, haya influido en el desarrollo de una nueva con cepción de los mismos, que busca definir sus estrategias de vida y encontrar en ellos regularidades (Grime,1974, 1977 y 1978 ; Whittaker y Goodman, 1979).

En el mismo sentido Margalef (1968) mantiene que la población debe verse más como un proceso que como un estado, y que a nivel de la comunidad se integran los procesos demográficos de las distintas especies, con es trategias de vida diferentes, pero no de forma aleatoria sino regularizadas por su incorporación en el proceso más general de la sucesión.

Con el presente trabajo se pretende comparar el comportamiento demográfico de dos especies de jaras ( $\mathrm{C} i s$ tus albidus y Cistus clusii), con el aporte de datos que ponen de manifiesto este proceso, y que permiten di ferenciar estrategias de vida y hacer predicciones de los cambios de vegetación.

AREA DE ESTUDIO

\section{LOCALIZACION GEOGRAFICA}

El área estudiada se encuentra al oeste de la provincia de Alicante, en una comarca caracterizada por grandes llanuras cultivadas, que separan afloramientos montañosos, generalmente desprovistos de arbolado, en los que dominan los matorrales. La parcela de estudio, for ma parte de la Sierra de Crevillente, situada en su sec tor noroeste; sus coordenadas U.T.M. son XH-859401. Tie ne una extensión de 15 ha y su cota máxima es de $530 \mathrm{~m}$.

\section{GEOLOGIA}

La bibliografía consultada (Azema, 1977) indica que el substrato está formado por brechas dolomíticas del Jurásico Inferior, en el dominio Subbético (subdivi sión dentro de las cordilleras Béticas). 
En cuanto a la estratigrafía, los estratos cali zos buzan sensiblemente al Noroeste, y en los puntos don de no afloran las calizas, existe Cuaternario (cantos, renas, arcillas). Por causa del clima y del substrato, existe un desarrollo acusado de Karts, siendo evidentes las formas de Lenar y Lapiaz.

\section{CLIMA}

Los datos de precipitación para el período de 1959-1972, tomados en la estación de Hondón de las Nie ves (situada a $2 \mathrm{~km}$ de la parcela) dan una precipitación media anual de $271.4 \mathrm{~mm}$; para el periodo de 1972-1979, datos tomados en la estación de Aspe (a $8 \mathrm{~km})$, la precí pitación media anual es de $270.5 \mathrm{~mm}$. Es de señalar que recientemente la sequía ha sido más acentuada; en 1978 la precipitación total anual fue de $159 \mathrm{~mm}$.

Aunque no se dispone de datos de temperaturas, pa rece lógico encuadrar a la zona en una situación intermedia entre las zonas Semi-Arida y Arida en que se divi de a la provincia.

\section{VEGETACION}

La zona de estudio estaría encuadrada dentro de la Alianza Rosmarino-Ericion Br.-Bl. 1930, y muy próxima a la asociación Fumano (ericoidis)-Stipetum tenacissimae $\mathrm{Br} .-\mathrm{Bl}$. et Bolòs (1957), recientemente tratada por BOLOS (1979).

La comunidad tiene aspecto de un herbazal-mato rral claro o medianamente denso, en el que el estrato ar bóreo de Pinus halepensis no es nunca denso, ni consigue gran altura. Bolòs (op.cit.) apunta que la aridez extre ma de la zona podría ocasionar actualmente en dicha co munidad, una sustitución o predominio de las especies $1 \bar{i}$ nearifolias sobre las planifolias, lo cual, dado que $C$. albidus es planifolia y C.clusii es linearifolia, aumen ta el interés de sus predicciones demográficas y su in terpretación posterior en relación con la sucesión . 
E1 empleo del método de regresión alométrica (Whittaker y Woodwell, 1969; Duvigneaud y col., 1977), proporciona la biomasa y producción de cada una de las fracciones en que se secciona la planta, y que en este caso fueron: hojas, ramas de uno y dos años, resto de ra mas y tronco, y frutos, referidas a las variables inde pendientes estimadas que son: el diámetro basal (tomado entre 0 y $3 \mathrm{~cm}$ ), la altura de la planta (distancia ver tical desde el suelo al punto más alto de la planta), y la longitud de la rama más larga (L.R.L.) que se define como el recorrido más largo que se puede hacer desde la base del tronco, siguiendo el contorno de la rama de ma yor longitud, hasta el extremo de êsta. Asimismo se dê terminó la edad por medio de los anillos de la base de tronco. Cada una de las fracciones fueron secadas a es tufa a $105^{\circ} \mathrm{C}$ hasta peso constante, y pesadas con una ba lanza cuya precisión es de $0.01 \mathrm{~g}$. Para los diámetros, la precisión fue de $0.1 \mathrm{~mm}$, mediante un pie de rey.

La estructura de la población se determinó mediante el estudio detallado de 43 parcelas de $2 \times 2 \mathrm{~m}$, distribuidas regularmente por la zona de estudio, en las que se realizaron dos censos. En el primero (Junio 1978) se marcaron todos los individuos de las dos especies y se tomaron las variables independientes, empleadas en la regresión alométrica. Un año después se comprobaron los supervivientes de la población inicial, así como el por centaje de ramas muertas respecto al total de la planta. En el segundo censo, se delimitaron 43 subparcelas de $50 \times 50 \mathrm{~cm}$, en las que se estudió con detalle la dinámi ca de la fase de plántula, que se definió como los indí viduos de menos de $3 \mathrm{~cm}$ de altura, con el fin de comple tar los datos de altas en la población y el éxito de la germinación en el campo. Todos los individuos censados se clasifican por regresión de la variable independiente L.R.L. en clases de tamaño-edad. Las clases de edad es tablecidas en la población corresponden a periodos de un año .

Los datos de reproducción provienen igualmente de la regresión alométrica, completada con el estudio de la capacidad germinativa de las semillas, que se determinó según las Reglas Internacionales para Ensayo de Se 
millas, de 1976 . La predación se estimó por observación directa de las señales y larvas de insectos, en todos los frutos recolectados (Bostock y Benton, 1979).

Obtenidos los datos de la estructura de la pobla ción, las tasas de supervivencia y natalidad, para cadā clase de tamaño-edad, se aplicó el modelo de la matriz de Leslie, que permite obtener una idea de la estabilidad y tendencia futura de la población (Usher, 1972).

\section{RESULTADOS Y DISCUSION}

\section{CENSOS DE LA POBLACION}

Los resultados globales de los censos realizados (tabla 1) muestran que no hay apenas variación en la den sidad de la población de ninguna de las especies, al pá sar un año. En los datos de dicha tabla no se toman en cuenta las plántulas, puesto que en 1978 no se estimó su población.

\begin{tabular}{|c|c|c|c|c|}
\hline & C E N S O DE 1978 & C E N S O & DE 1979 \\
\cline { 2 - 5 } & C.albidus & C.clusii & C.albidus & C.clusii \\
\hline$N^{\circ}$ INDIVIDUOS & 189 & 255 & 187 & 254 \\
\hline$N^{\circ}$ INDIV/M 2 & 1.09 & 1.48 & 1.08 & 1.47 \\
\hline
\end{tabular}

TABLA 1 . Resultados globales de los censos reali zados en la zona de estudio en Junio 1978 y Jü nio 1979 . Los datos consideran sólo los individuos mayores de $3 \mathrm{~cm}$.

Summary of data from two census, June 78 and June 79 . 
Uno de los aspectos más interesantes de la demo grafía vegetal es la peculiaridad de las plantas de su frir pérdidas de sólo parte de su biomasa vegetativa. És te hecho se manifiesta como muerte de parte de un indivi duo censado que, sin embargo, permanece como tal indivi duo en la población.

Este tipo de mortalidad que hemos denominado "parcial" parece tener su causa (Zimmerman y Brown,1971) en un fenómeno fisiológico por el que algunos arbustos, incapaces de mantener en contínuo aumento el sistema de haces conductores, resuelven eliminar parte de sus rami ficaciones, originando asî una disminución de su biomasa aérea .

Las figs. 1 y 2 muestran la variación de la tasa de mortalidad parcial encontrada en ambas especies con re lación a la clase de tamaño-edad; como se ve en los dos casos, dicha tasa aumenta con la clase tamaño-edad, es de cir, la mortalidad parcial afecta más a los individuos a dultos que a los jóvenes. Este comportamiento parece conn cordar con la interpretaciôn dada por Zimmerman y Brown (op.cit.).

A1 tipo de mortalidad que afecta a todo el indi viduo la denominamos "total"; en este caso es todo el in dividuo el que causa baja en la población. Las figuras $\overline{3}$ y 4 muestran la distribución de la tasa de mortalidad to tal respecto a las clases de tamaño-edad. Se observa que en el caso de C.Clusii (fig.3) la tasa de mortalidad to tal es independiente de la edad, afectando por igual a in dividuos de toda la población, mientras que C.a.bidus (fig.4) parece tener un comportamiento distinto, afectan do más a los individuos más adultos de la población, de forma semejante a la hallada en especies de Pinus strobus y Acer rubmu por Hett y Loucks (1968).

Ante este fenómeno propio de la vegetación,y al considerar que tanto la mortalidad "parcial" como la "to tal" suponen una pérdida de biomasa para la población, se ha optado por establecer las tasas de mortalidad para ca da clase de tamaño-edad, tomando como unidad la biomasa (Biomasa perdida por la clase i/Biomasa total de la cla- 


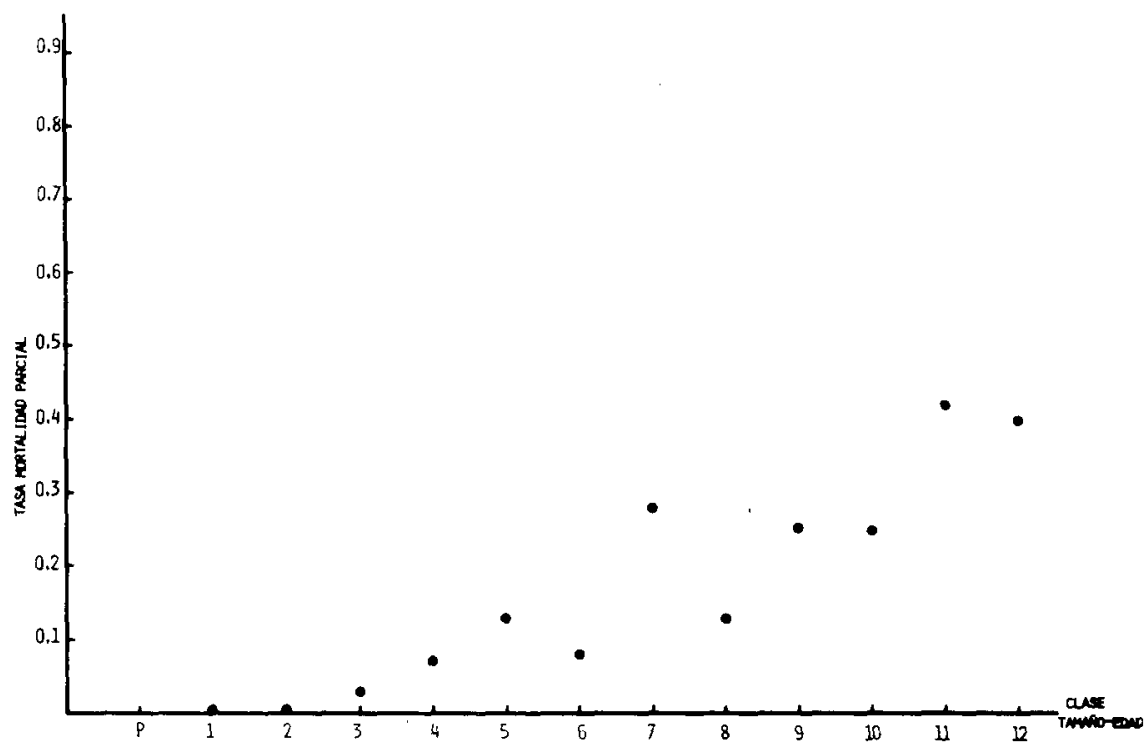

Figura 1 . Variación de la tasa de mortalidad parcial con relacion a la clase tamaño-edad en Cistus clusit.

Partial mortality rate variation in relation to size-age class, in cistus clusii.

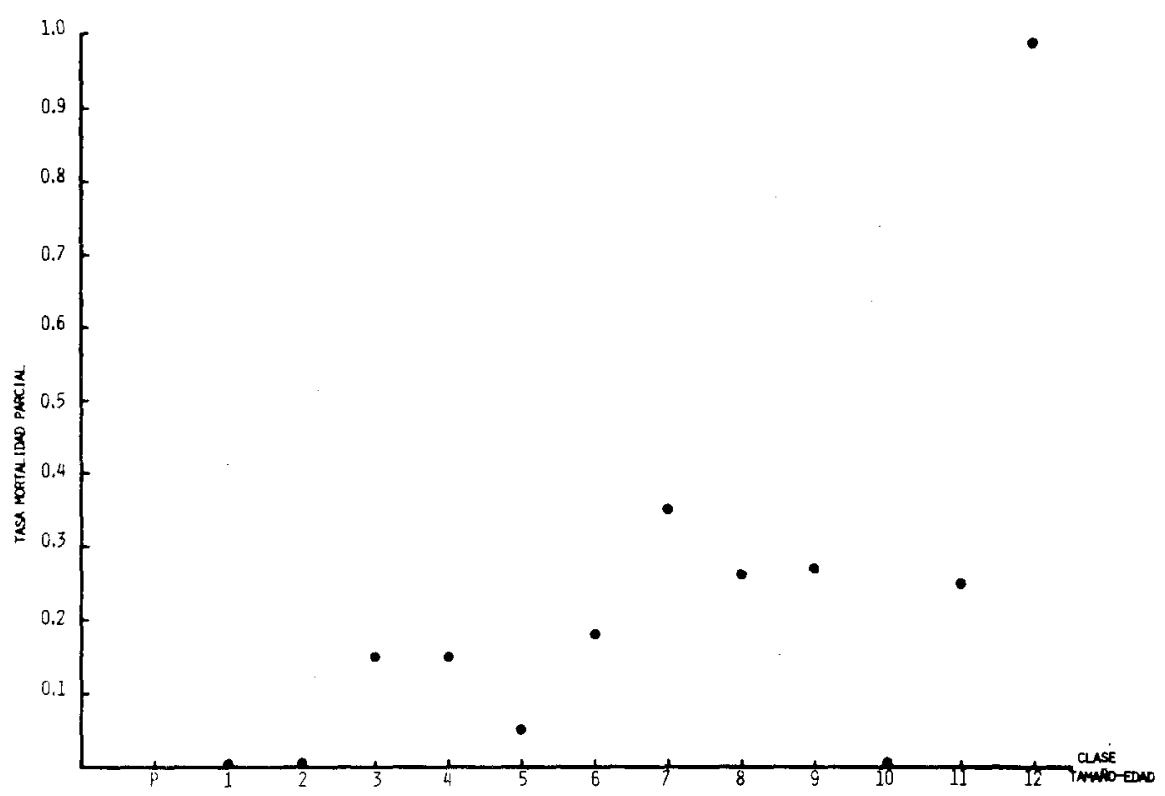

Figura 2. Variacion de 1 a tasa de mortalidad parcial con relación a la clase tamano-edad en Cistus albidue.

Partial mortality rate variation in relation to size-age class, in cistus atbidus. 


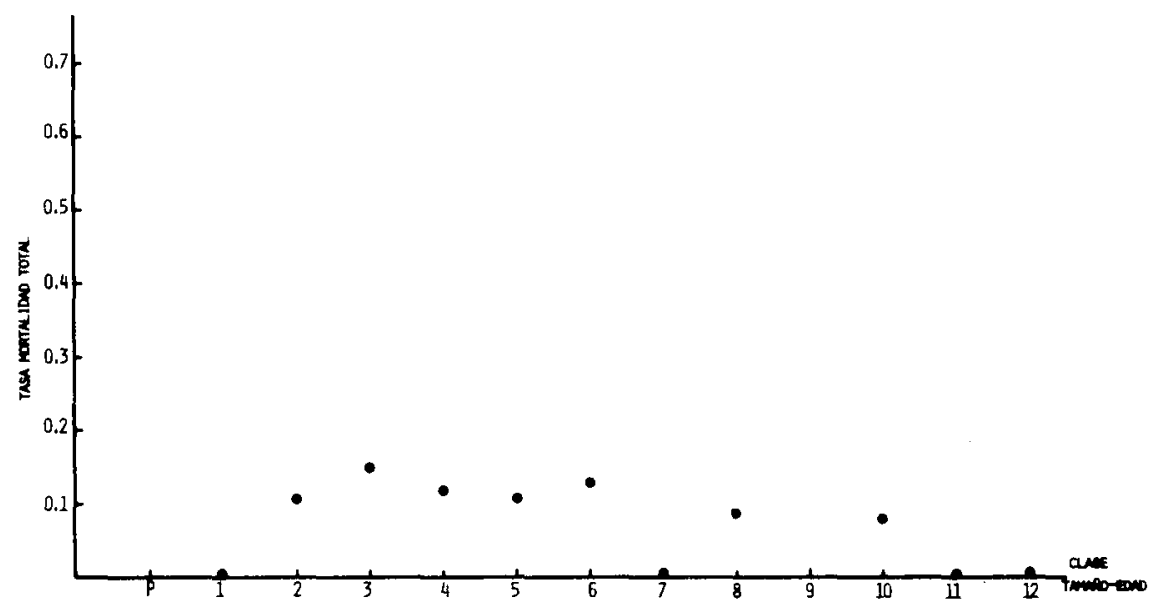

Figura 3 . Variación de la tasa de mortalidad total con relación a la clase tamaño-edad en Ciatus cluati.

Total mortality rate variation in relation to size-age class, in cistus olusiz.

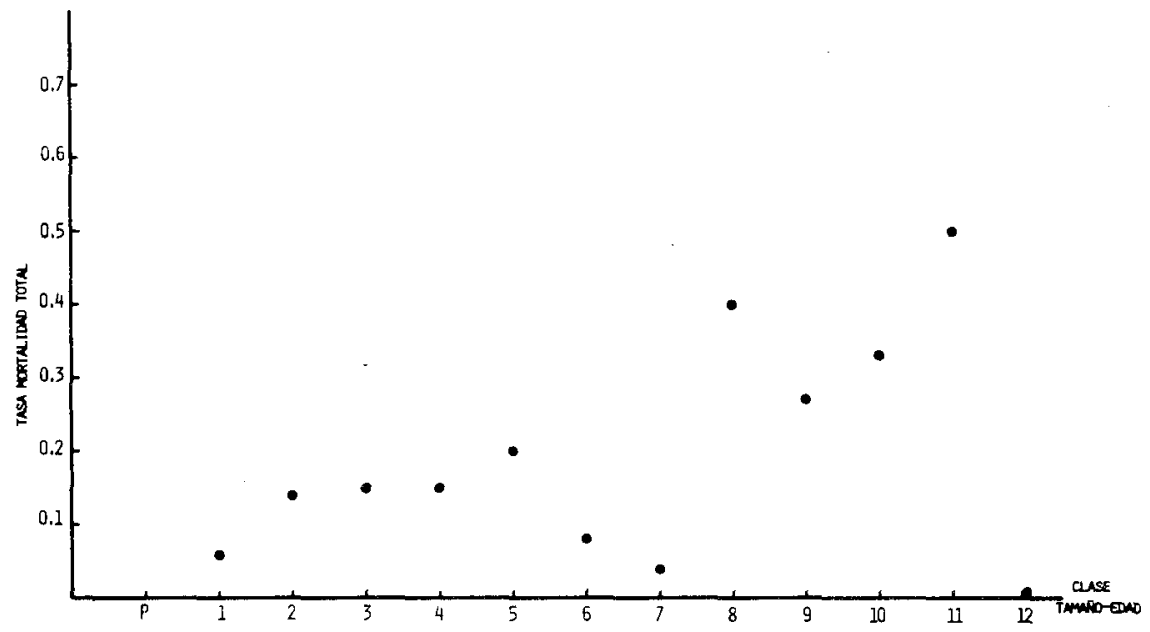

Figura 4 . Variación de la tasa de mortalidad total con relación a la clase tamaño-edad en Cistus albidus.

Total mortality rate variation in relation to size-age class, in ctistis albidue. 
se i).E1 efecto de esta tasa de mortalidad para cada clase de tamaño-edad, se refleja en las curvas de superviven cia de ambas especies (fig.5), donde se observa una altā mortalidad en las plántulas en las dos especies, y cierta diferencia a partir de este punto, que sugiere una mayor independencia entre edad y mortalidad en C.clusii.

Una forma de explorar la dependencia de la super vivencia respecto a la edad, aspecto que está en la basé de la distinción de tipos de curvas de supervivencia, es el propuesto por Hett y Loucks (1976) por medio del ajus te a expresiones de tipo exponencial o potencial, según se presuma o no la existencia de relación entre ambas va riables.

Para las jaras se han encontado mejores ajustes a funciones exponenciales (tabla 2) que a potenciales, 10 que hablaría a favor de aceptar una independencia de la mortalidad respecto a la edad, más patente en C.aZbidus que en C.clusii.

Dado que en trabajos como el anteriormente citado, la estructura sincrónica de edades se proyecta como diacrónica, ha parecido de interés comparar para los Cis tus, aunque de vida mucho más corta que las especies ar bóreas por ellos tratadas, tal procedimiento con el segui do en este estudio que sí contiene, aunque sólo para un año, una estima de las variaciones diacrónicas. En la ta bla 2 puede apreciarse que los ajustes son peores si se parte de un sólo análisis estructural.

\section{NATALIDAD}

Uno de los apartados previos al presente trabajo, permite obtener una estima del esfuerzo reproductivo de las especies, tal como lo definen Gadgil y Solbrig (1972) así como la eficiencia de dicho esfuerzo (número de plán tulas final/número total de semillas producidas), que ré sume en cierta forma el \% de semillas perdidas por preda ción de insectos, las no germinadas (análisis de germiná ción) y las que pasan al "banco de semillas del suelo " (Paterson et al., 1976; Harper,op.cit; Turkington et al. 1979 ). 


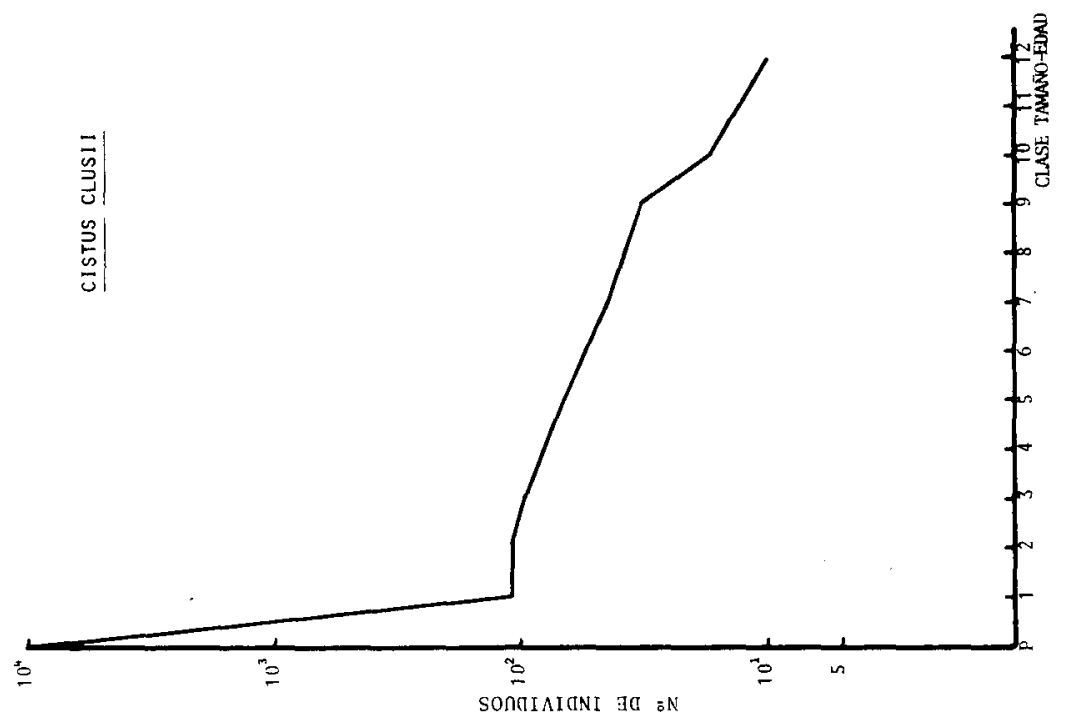




\begin{tabular}{|l|r|r|r|r|}
\hline & \multicolumn{2}{|c|}{ MOD.DIACRONICO } & \multicolumn{2}{c|}{ MOD. SINCRONICO } \\
\cline { 2 - 5 } & Potencial & Exponencial & Potencial & Exponencial \\
\hline C. albidus & $-0.87 *$ & $-0.97 *$ & $-0.84 *$ & $-0.84 *$ \\
\hline C. clusii & $-0.92 *$ & $-0.96 *$ & -0.54 & -0.54 \\
\hline
\end{tabular}

Tabla 2 . Valor de los coef, de correlación $r$ encontrados para el ajuste del $\mathrm{n}^{\circ}$ de indiv. para cada clase tamaño-edad, a expresiones de ti po exponencial y potencial, tanto para el mod.diacrónico como para el mod.sincrónico. (*) Indican valores de $\mathrm{r}$ significativos con una seguridad $\mathrm{P}<0.001$

Correlation coefficients $(r)$ for the fit between the number of plants for size-age class, and two kinds of expressions, exponential and potential, for synchronous as well as diachronous projections. (*) Corr.coeff. significant for $\mathrm{p}<0.001$.

La tabla 3 resume estos cálculos y se observa un mayor Indice de eficiencia para C.albidus, a pesar de que es la especie más predada ( $31 \%)$, con menor \% de germinación (19\%) y con menor producción de semillas; la causa de esta mayor eficiencia radica en la tasa de superviven cia de las plántulas, que compensa a la especie de la de ficiencia en los otros aspectos.

Sobre la incidencia de la predación, no se tienen datos de especies próximas a las estudiadas, y los valo res encontrados están comprendidos entre los que dan Bos tock y Benton (1979), para Cirsium arvense (43\%), Tussilago farfara (21\%) y Taraxarum officinale (2\%).

E1 peso medio de la semilla (C.albidus $0.98 \mathrm{mg}$ y C.clusii $0.29 \mathrm{mg}$ ) es similar al de otras especies acompa ñantes en el matorral (Madueño (1973), cita para Rosmari nus officinalis $1.03 \mathrm{mg}$, y para Thymus vulgaris $0.26 \mathrm{mg}$ ). 


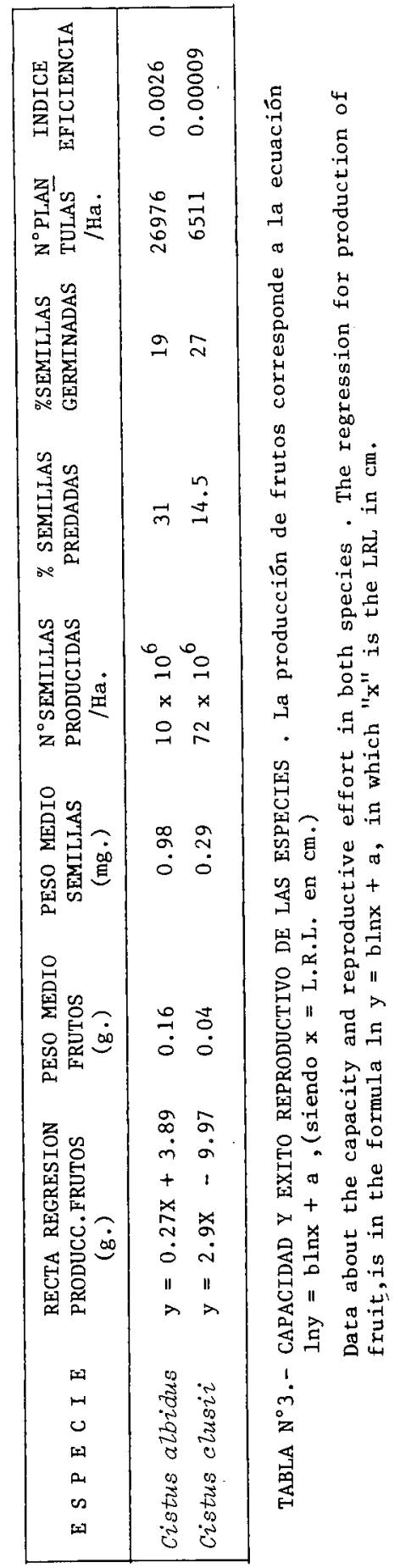

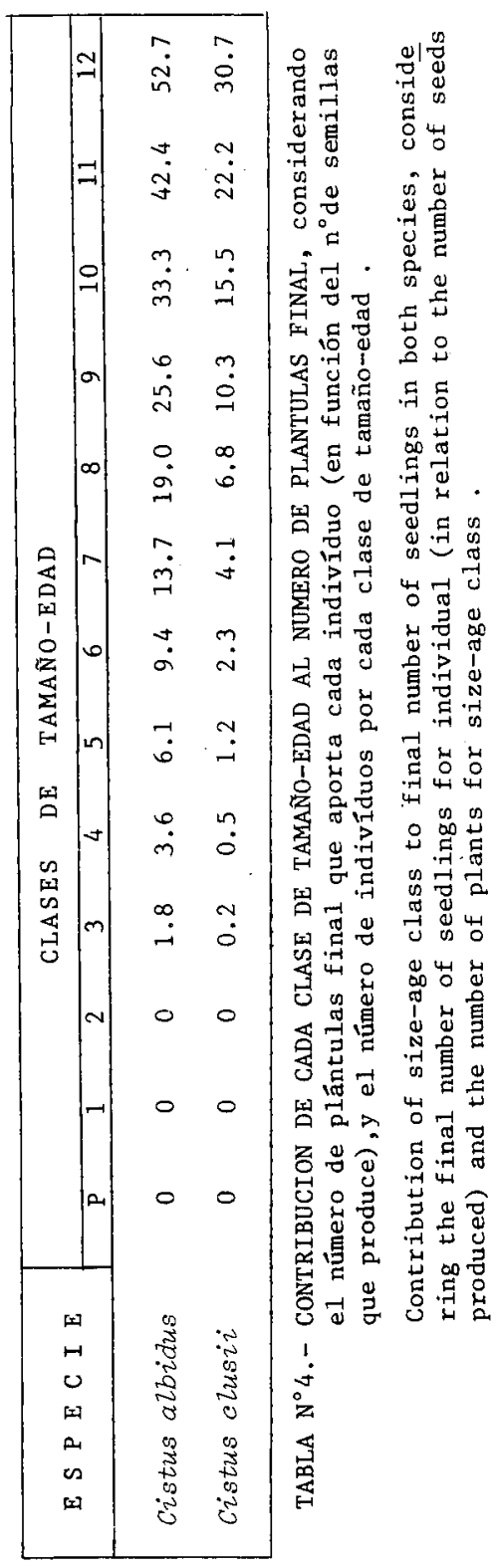


En cuanto a la capacidad germinativa, datos pro porcionados por Catalán (1978), dan para Rosmarinus off $\bar{i}$ cinalis 36-40\% y Quercus coccifera 60-80\%, 10 que sitúa a las especies de Cistus estudiadas, en ligera desventaja para ocupar el espacio, frente a estas asíduas acompañan tes.

La contribución de cada clase de tamaño-edad a la natalidad final de la población, se muestra en la tabla 4. Aceptamos que la capacidad germinativa de las semillas no está afectada por la edad de la planta madre, aceptación hecha por múltiples autores (Okusanya, 1979; Huiskes, 1979) . Se considera que la aportación de las clases de plántula, uno y dos años es nula, puesto que no se han ob servado en ellas individuos con frutos.

E1 esfuerzo reproductivo es una función de la bio masa total aérea y de las condiciones ambientales a que está sometida la población (Gadgil y Solbrig op.cit.); su expresión en \% de producción (peso seco) de frutos, res pecto al \% de producción de hojas y \% de producción de ramas $\bar{y}$ tronco, se muestra en las figuras números 6 y 7 . Se ob serva que en $C . a$. bidus el $\%$ de frutos producidos se man tiene entre 13-19\%, y no varía apenas con la edad. En $C$. clusii, el \% dedicado a frutos varía ostensiblemente con la edad, siendo $14 \%$ para los más jôvenes y 11 egando al $37 \%$ en los adu1tos. En ambos casos el aumento del \% de fru tos producidos con la edad, se efectúa a expensas de la producción de hojas.

Datos similares de producción de frutos a los de C.albidus los dan Bostock y Benton (op.cit.) para Achi Zlea millefolium 19-24\%; Taraxam officinale 20-29\% y ssilago farfara 20-32\%. C.clusii parece distinguirse por ser una especie con poca eficiencia, que intenta compensarse con un gran esfuerzo reproductivo.

\section{MODELO PREDICTIVO DE LA POBLACION}

Con una población inicia1 con cierta distribución de clases de tamaño-edad, al aplicar sus propias tasas de natalidad y fertilidad, se obtiene la composición en el siguiente período de tiempo. Podemos proyectar en el tiempo cualquier composición actual de la población, me- 

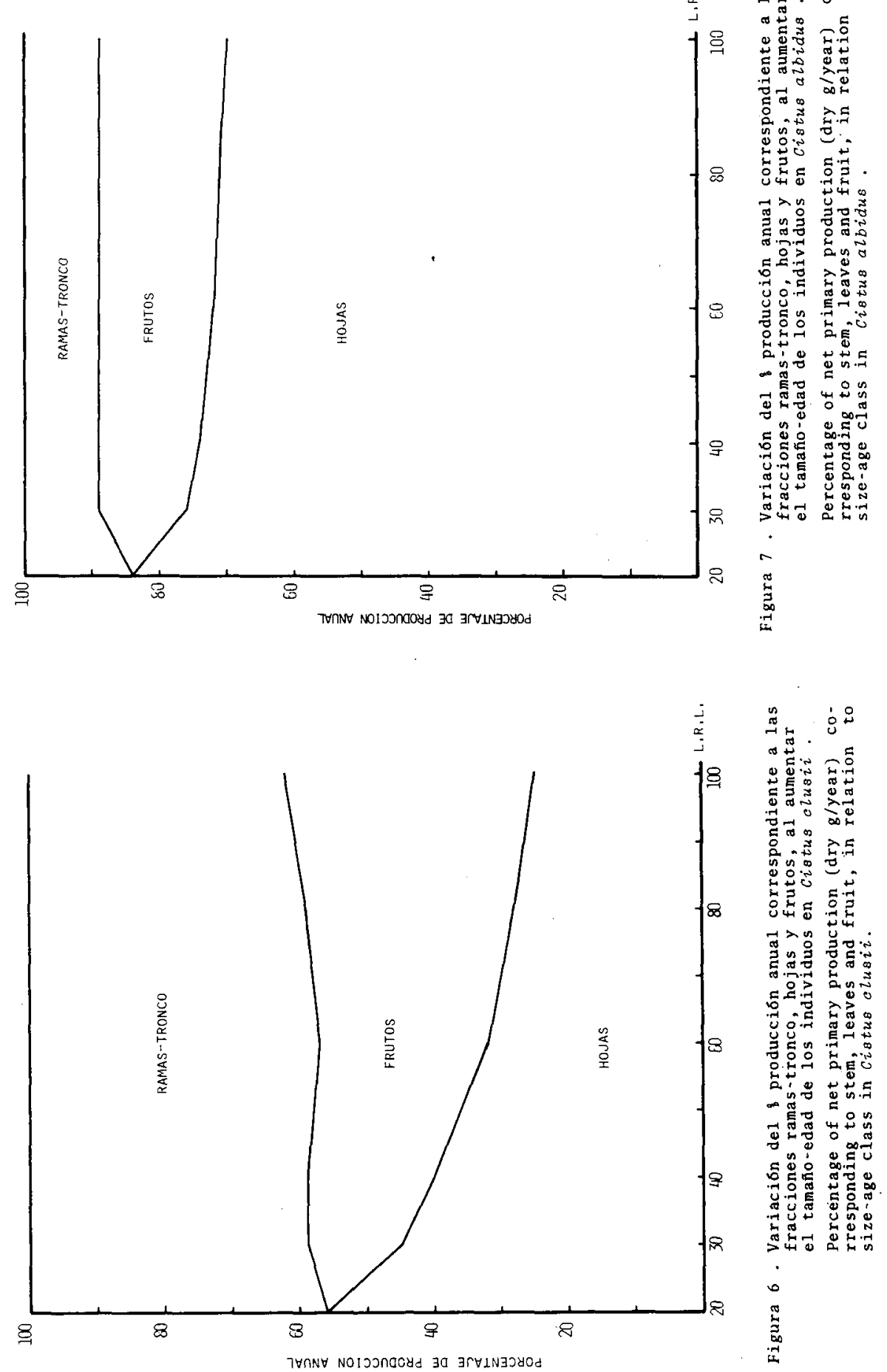
diante un proceso iterativo (modelo matricial de LESLIE) y suponer que dichas tasas se mantienen constantes. Una población que evoluciona así, alcanza asintóticamente una distribución estable (Margalef, 1974) . Esta estructura estable de la población, corresponde en términos matricia les al vector propio $\lambda$, que va asociado al valor propio n de la Matriz de Lesīie, que debe ser positivo y máximo o igual a otros valores propios de la matriz (Legendre y Legendre, 1979) . El cálculo de los autovalores y autovec tores de las matrices de ambas especies (Tablas 5 y 6) se efectuó en la terminal DCT-2000 del Centro de Cálculo de la Universidad de Alicante, con un programa Fortran IV denominado VECTOR, que utiliza la Subrutina AUTOV que fi gura en Davis (1971).

Los valores de $\lambda$ encontrados (tabla 7) indican que ambas poblaciones tienen una tasa de crecimiento ins tantáneo ( $r$ ) positiva. Werner y Caswell(op.cit), en Dipsa cus sylvestris, encuentran un amplio espectro de valores de $\lambda$, en función de la vegetación característica del cam po de estudio. Sarukhan y Gadgil (op.cit) dan resultados igualmente variables para tres especies de Ranunculus. Esta gran variabilidad del valor de $\underline{\lambda}$ se atribuye a que viven en situaciones desequilibradas. Comparativamente, los valores de $\lambda$ encontrados sugieren que las especies es tudiadas no estắn demasiado lejos de la situación estacio naria.

La estructura de la población, obtenida por el mo delo de Leslie, se representa en las figs. 8 y 9 ; se ob serva que para $C$. Clusii hay una acumulación de indiv $\bar{i}$ duos mayores de 12 años, 10 que concuerda con la curva de supervivencia y hace pensar que la población de C.clusiz todavía no ha llegado a su máxima madurez. En las figs. 8 y 9 se representa también la estructura de la población hallada en el censo de 1979, por la que se muestra eldes ajuste existente. E1 grado de identidad entre ambas es tructuras (observada y estable) se valora mediante el in dice de desemejanza $\Delta$ (Keyfitz y Flieger, 1975), que es la suma de las diferencias positivas en porcentaje. Los valores hallados para $\triangle$ (tabla 7 ), indican que ambas espe cies están fuertemente alejadas de la distribución está ble de edades y es mayor el alejamiento en C.clusii. 


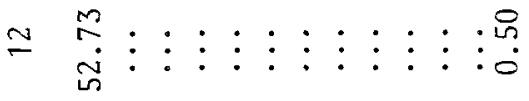

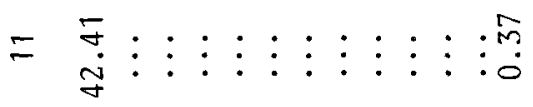

$$
\begin{aligned}
& \circ \underset{\text { 命 }}{m}: \vdots \vdots \vdots \vdots \vdots \vdots \vdots: \stackrel{\circ}{0}:
\end{aligned}
$$

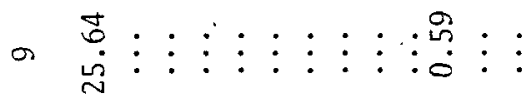

$$
\begin{aligned}
& \underset{\sim}{\omega} \\
& \text { 㟔 } \\
& \infty \stackrel{8}{\circ}: \vdots \vdots \vdots \vdots \vdots \vdots \stackrel{\circ}{\circ}: \vdots \vdots
\end{aligned}
$$

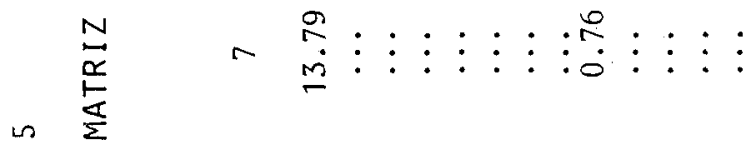

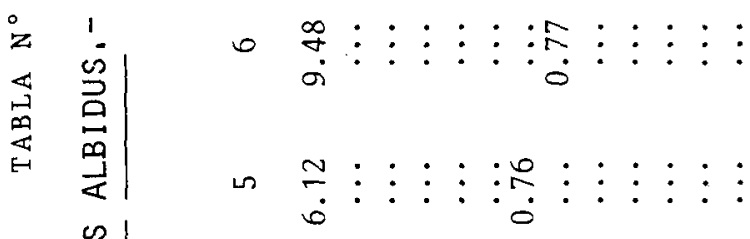

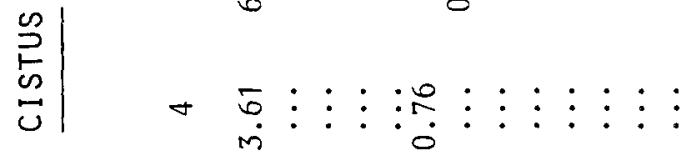

$$
\begin{aligned}
& \text { m } \stackrel{\circ}{\infty}: \vdots \stackrel{0}{\sim}: \vdots: \vdots \vdots \vdots \vdots \\
& \text { ^ } \circ \vdots:_{0}^{\infty}: \vdots \vdots \vdots: \vdots \vdots \vdots \vdots
\end{aligned}
$$

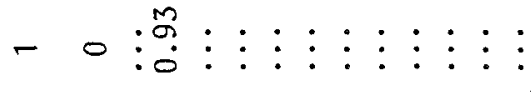

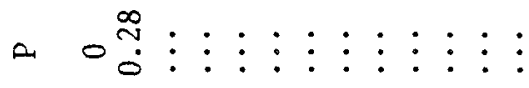

$$
\begin{aligned}
& \text { a - NMt L ONODOO= }
\end{aligned}
$$




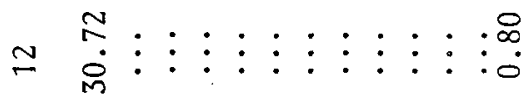

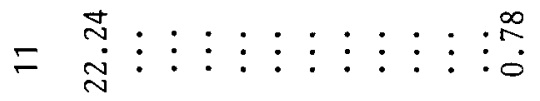

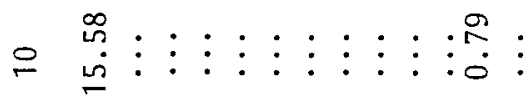

$$
\begin{aligned}
& \text { ๙ } \\
& \infty \underset{; \infty}{\infty}: \vdots: \vdots: \vdots \vdots: \infty \vdots: \vdots:
\end{aligned}
$$

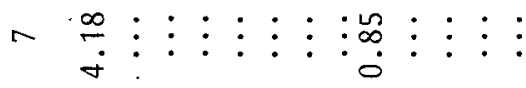

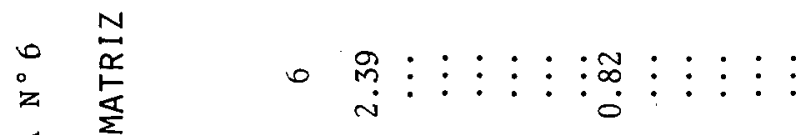

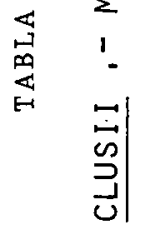

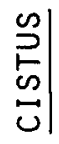

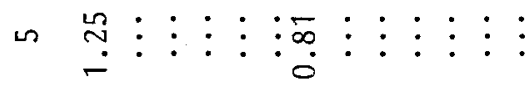

$$
\begin{aligned}
& +\quad \text { 足: }: \vdots::_{0}^{\infty}: \vdots: \vdots: \vdots: \vdots \\
& \text { m } \underset{0}{\sim}::: \stackrel{\infty}{\infty}: \vdots: \vdots: \vdots: \vdots: \\
& \sim \circ: \vdots: \infty \vdots: \vdots: \vdots: \vdots: \vdots: \\
& \text { - о : } \\
& \text { ค о } \div: \vdots: \vdots: \vdots \vdots: \vdots \vdots:
\end{aligned}
$$

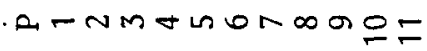




\begin{tabular}{|c|c|c|}
\hline & C.albidus & C.clusii \\
\hline$\lambda$ & 1.298 & 1.104 \\
\hline $\mathrm{r}$ & 0.260 & 0.098 \\
\hline $\mathrm{n}$ & 0.959 & 0.985 \\
& 0.207 & 0.098 \\
& 0.149 & 0.089 \\
& 0.098 & 0.071 \\
& 0.058 & 0.053 \\
& 0.035 & 0.041 \\
& 0.020 & 0.030 \\
& 0.012 & 0.022 \\
& 0.007 & 0.017 \\
& 0.003 & 0.013 \\
& 0.001 & 0.006 \\
& 0.001 & 0.005 \\
& 0.000 & 0.012 \\
\hline
\end{tabular}

TABLA $N^{\circ}$ 7. - Valores del autovalor $(\lambda)$, de la matriz de Leslie, del autovector asociado (n) de la tasa instantánea de crecimiento $(r=\ln \lambda)$ $y$ del Indice de desemejanza $\Delta$, para ambas especies.

Values corresponding to the eigenvalues $(\lambda)$ of the Leslie matrix, to the associated eigenvectors $(n)$, to the instantaneous growth rates, and to the dissimilarity in $\operatorname{dex}(\Delta)$, for both species. 

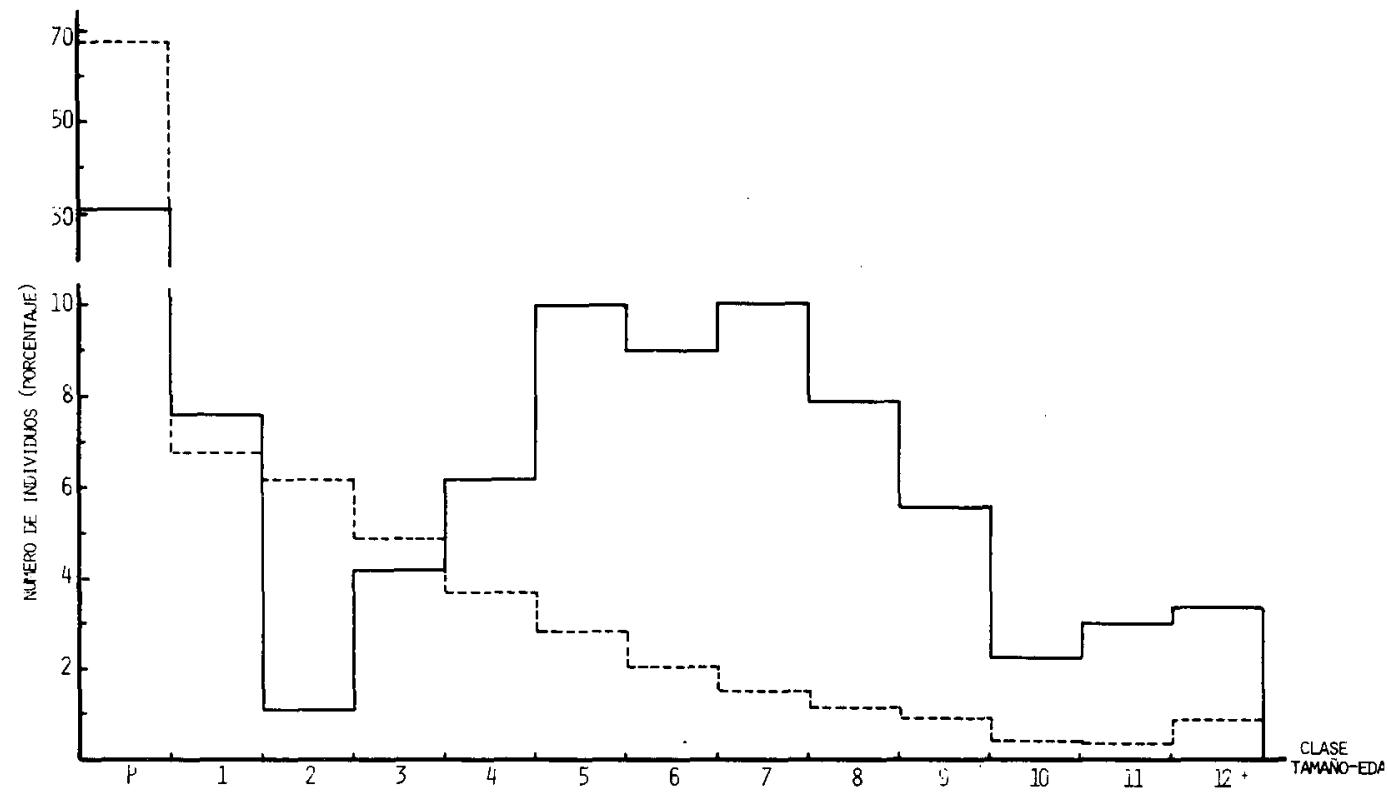

Figura 8 . Histograma continuo de comparación por clases tamaño-edad entre la población observada (-) y el modelo de población estable obtenido por la matriz de Leslie (--) en Cistus clusii.

Size-age diagrams for the current structure of population ( - ) and the stable one obtained from Leslie matrix (-..) in Cistus cluaii.

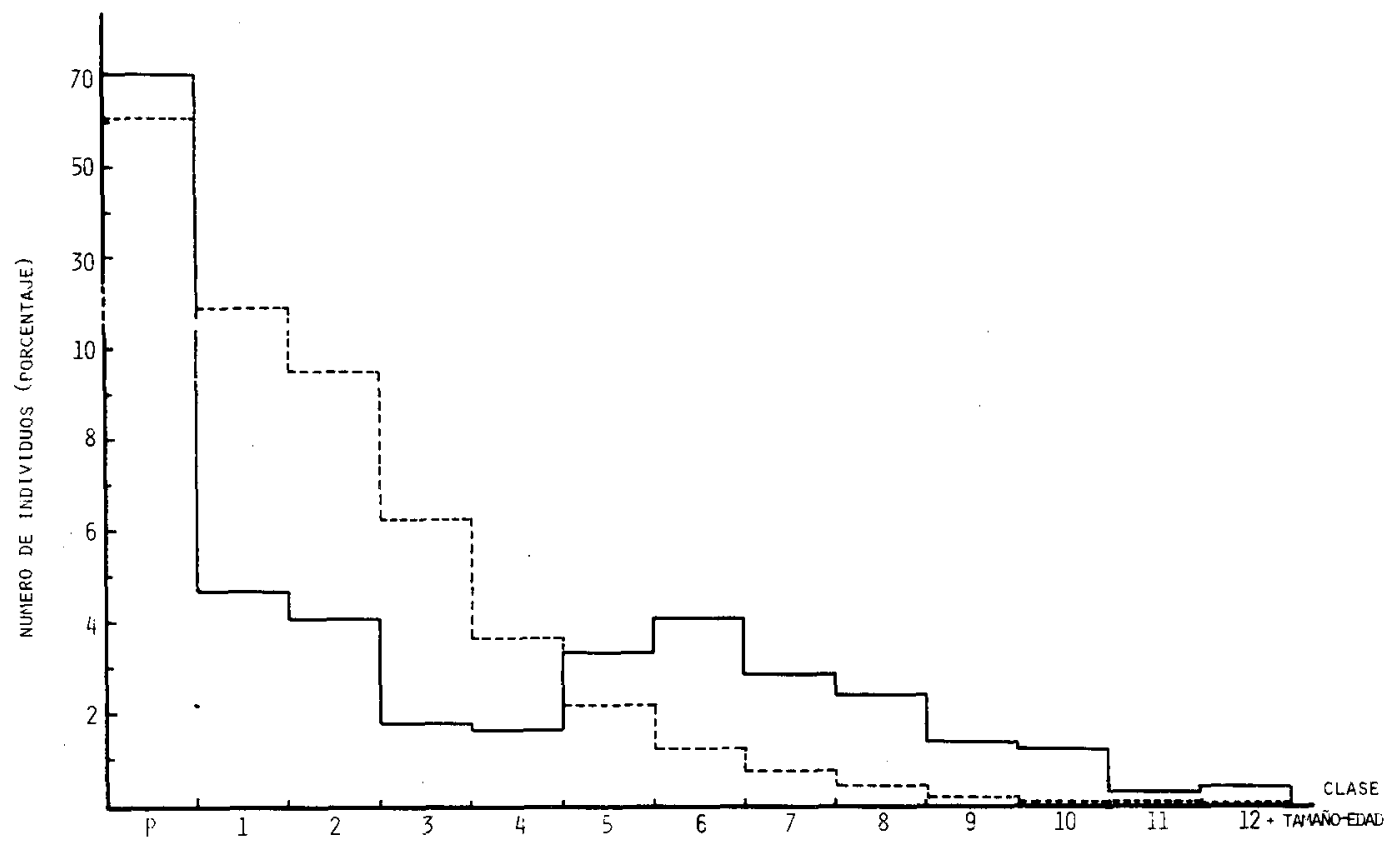

Figura 9 . Histograma contínuo de comparación por clases tamaño-edad entre la población observada (-) y el modelo de población estable obtenido por la matriz de Leslie (...) en Cistus albidus.

Size-age diagrams for the current structure of population (-) and the stable one obtained from leslie matrix (-.-) in Cistus albidus. 
Con los resultados obtenidos, es difícil apoyar la idea de una sustitución de especies planifolias por li nearifolias (Bolos,op. cit) en la zona estudiada, si bien podemos aproximar los resultados a una hipotética sustitución, si interpretamos que la población de C.albidus es tâ en su máximo grado de madurez (la longevidad actual es la máxima que alcanza la especie) y que su desajuste res pecto del modelo estable, se debe a que ha iniciado su re troceso. Respecto a C.clusii, su actual desajuste podría deberse por el contrario a que está en fase de desarrollo sin haber alcanzado la población la madurez máxima, a fal ta de individuos mäs adultos que, de estar presentes, pó drían cambiar totalmente las tasas de reproducción, y a terar el valor actual de $\lambda$.

\section{RESUMEN}

Se estudia la demografía de dos especies de $C i s$ tus, Cistus albidus y Cistus clusii, muy abundantes en el matorral mediterráneo, en una parcela de $15 \mathrm{Ha}$. en la Sierra de Crevillente (Alicante). El substrato es calcáreo y la vegetación puede considerarse perteneciente a la asociación Fumano (ericoidis)-Stipetum tenacissimae Br.Bl. et Bolós (1957).

El principal interés de este tipo de estudio es la posibilidad de hacer predicciones sobre el futuro de ambas poblaciones en relación con cambios hipotéticos en la sucesión.

Se censaron en dos ocasiones, con un año de inter valo, 43 parcelas de $2 \times 2 \mathrm{~m}$. y distribuidas uniformemen te. La densidad (C.albidus 1.09 y C.clusii 1.48 indivi duos por metro cuadradol y estructura de tamaño-edad, se obtuvo cada vez. Es posible distinguir dos clases de mor talidad, parcial y total, que muestran distinta relación con el tamaño-edad en las dos especies.

Con las dos tasas de mortalidad combinadas, se ob tuvieron curvas de supervivencia. En ellas, y para las dos especies, destaca una mortalidad muy alta para las - 
plántulas. Se ensaya el ajuste de estas curvas a expre siones exponenciales y potenciales. El mejor ajuste al modelo exponencial indicaría falta de dependencia entre edad y mortalidad, particularmente en C.albidus. Además, la proyección de las estructuras de edades sincrónicas de las poblaciones como diacrónicas, da peores ajustes en ambos modelos.

Se ha evaluado el esfuerzo reproductor como el 13-19\% de la producción primaria neta en C.albidus y el $14-37 \%$ en C.clusii . Esto puede considerarse como un ca rácter "competitivo" de ambas especies, que estaría com pensado por la predación de frutos ( 31 y $14 \%$ en peso res pectivamente en C.albidus y C.clusii), tasa de germinación de semillas baja (19 y $27 \%$ ) y gran mortalidad de plántulas $(72$ y $89 \%)$. El resultado es una mayor eficien cia de C.albidus (0.0026) que en C.clusii (0.00009).

Se utilizó la matriz de Leslie en la predicción de futuras estructuras de las poblaciones. El autovalor fue de 1.298 para C.albidus y de 1.104 en C.clusii, y los valores correspondientes de $r$ son 0.26 y 0.098 , el segundo de los cuales es muy próximo al de un estado es tacionario. El desajuste existente entre la estructura actual de la población y la estructura estable obtenida por el autovector asociado al autovalor de la matriz de Leslie puede expresarse por medio del índice de disimilaridad. Los valores de dicho índice son $\Delta=20.72$ para C.albidus y $\Delta=43.45$ en C.clusii, más alejada ésta última especie del modelo estable.

\section{SUMMARY}

The demography of two species of Cistus, C.albi dus and C.clusii, very abundant in the Mediterranean shruby vegetation, was studied in a $15 \mathrm{Ha}$ plot in Sierra de Crevillente (Alicante). The bedrock is calcareous and the vegetation can be classified as belonging to the association Fumano(ericoidis)-Stipetum tenacissimae $\mathrm{Br}$. Bl. et Bolós (1957).

The main interest of this kind of study is the possibility of making predictions about the future of - 
both populations in relation to hipothetical changes in succession.

Forty three plots of $2 \times 2 \mathrm{~m}$ uniformely distributed were censused for two species twice within a year's in terval. The density (C.albidus 1.09 individual per squa re meter, and C.clusii 1.48 in sq.m.) and size-age struc ture were obtained each time. It is possible to distinguish two kinds of mortality, parcial and total, that show different relation with the size-age in the two species.

Survivorship curves were obtained with the two mortality rates combined. The curves show a very high mortality in plantules in both species. The fit of this curve to exponential and potential expressions was tried. The best $f$ it to the exponential model would indicate lack of dependence between age and mortality, particulary in C.albidus. Moreover, the projection of synchronous sizeage structures of populations as diachronous ones give a poorer fit to both models.

The reproductive effort was evaluated as the 13$19 \%$ of net primary production in C.aZbidus and $14-37 \%$ in C.clusii. This can be considered as a "competitive" cha racteristic of both species that must compensate predation on fruits ( 31 and $14 \%$ in weigth respectively in $C$. albidus and C.clusii), low germination rate of seeds (19 and 27\%) and great mortality of plantules (72 and $89 \%$ ). The result is a great efficience of C.albidus (0.0026) that in C.clusii (0.00009).

Leslie matrix was used in the prediction of futu re structures of populations. The eigenvalue was $1.29 \overline{8}$ for C.aZbidus and 1.104 in C.clusii and the corresponding values for $r$ was 0.26 and 0.098 , the first of them very close to the stationary state. The disagreement between the present structure of population and the stable one ob tained by the eigenvectors associated to the eigenvalue of the Leslie matrix, can be expressed by the dissimilarity index. The values was $\Delta=20.72$ for C.aZbidus and $\Delta=43.45$ in C.clusii more far the last of the stable mode1 . 


\section{BIBLIOGRAFIA}

AZEMA, J. 1977 . Etude geologique des zones externs des Cordizzeres Betiques aux confins des Provinces d'Alicante et de Murcie (Espagne). Thèse Doctoral, 393 pp. Université de Paris VI.Paris

Bolos, 0.de 1979 . De vegetatione valentina. III. ButZZ. Inst. Cat. Hist.Nat, 44 (Sec.Bot) 3:65-76.

BOSTOCK, S.J. y BENTON, R.A. 1979 . The reproductive strategies of five perennial compositae . J.Ecol.67,1:91-107.

CASWELL, H. y WERNER, P.A. 1978 . Transient behavior and life history analysis of teasel (Dipsacus sylvestris Huds) . Ecology, $59,1: 53-66$.

CATALAN, G. 1978 . Semillas de árboles y arbustos forestales . IC $\underline{0}$ NA . Monografia, 17 . Ministerio de Agricultura .

DAVIS, R.G. 1971 . Computer Programing in Quantitative Biology. Academic Press . London and New York.

DUVIGNEAUD, P., KESTEMONT, P., TIMPERMAN, J. y MONIQUET, J.C. 1977. La Hetraie Ardennaise a Festuca altissima a Mirwart. Biomasse et Productivite primaire . En P.Duvigneaud y P.Kestemont (eds.) Productivité biologique en Belgique . SCOPE. Tr.Sec.belge du P.B.I.

GADGIL, M.G. y SOLBRING, O.T. 1972 . The concept of r-and k-Selec tion : Evidence from wild flowers theoretical considerations . Amer. Natur. Vol.106, $\mathrm{n}^{\circ} 947,14-31$.

GRIME, J.P. 1974 . Vegetation classificacion by reference to stra tegies . Nature, Vo1.250, 26-3.

GRIME, J.P. 1977 . Evidence for the existence of three primary strategies in plants and its relevance to ecological and evolu tionary theory. Amer. Natur. 111:1169-1194.

GRIME, J.P. 1978 . Interpretation of small-scale patterns in the distribution of plant species in space and time. En Freysen y Woldenlorg (Eds.). Stmicture and Functioning of Plant Popula tions . North-Holland Publishing Company, Amsterdam .

HARPER, J.L. 1977 . Population biology of plants. Academic Press New York .

HETT, J.M. y LOUCKS, O.L. 1968 . Application of lifetable analyses to tree seedlings in Quetico Provincial Park, Ontario . For. Chron. 44:29-32.

HETT, J.M. y LOUCKS, O.L. 1976 . Age structure models of balsam fir and eastern hemlock. J.Eco Z. 64,3:1029-1045.

HUBBELL, S.P. y WERNER, P.A. 1979 . On measuring the intrinsic ra te of increase of populations with heterogeneus life histories. Amer.Natur. Vo1.113, $\mathrm{n}^{\circ} 2,277-293$. 
HUISKES, A.H.L. 1979 . Biological flora of the British Isles, Amophiza arenaria (L.) Link. J.Ecol. 67,1:363-383.

KEYFITZ, N. y FLIEGER, W. 1975 . Demografla : Métodos Estadisticas Ed.Marymar. Buenos Aires.

LEGENDRE, L. y LEGENDRE, P. 1979 . Ecologie Numerique, Tome II, Co llection d'Ecologie $\mathrm{n}^{\circ} 13$. Edit. Masson.

MADUEÑo, M. 1973 . Cultivo de plantas medicinales. Ministerio de Agricultura . Madrid .

MARGALEF, R. 1968 . Perspectives in Ecological Theory. The Univer sity of Chicago Press.

MARGALEF, F. 1974 . Ecologia . Ed.Omega . Barcelona .

OKUSANYA, O.T. 1979 . An experimental investigation into the ecology of some maritime cliff species. II . Germination studies. J.Ecol. 67, 1:293-305.

PATERSON, J.G., BOYD, W.J.R. y GOODCHILD, N.A. 1976 . Effect of temperature and depth of buñal on the persistance of seed of Avena fatua L. in Western Australia . J.App L.Eco Z.13,3:841-849.

REGLAS INTERNACIONALES PARA ENSAYO DE SEMILLAS 1976 . Ministerio de Agricultura . Madrid .

SARUKHAN, J. y HARPER, J.L. 1973. Studies on plant demography : Rannunculus repens L., R.bulbosus L. and R.acris L. I. Population flux and survivorship . J.Ecol. 61,3:675-716.

SARUKHAN, J. y GADGIL, M. 1974 . Studies on plant demography: Ran nunculus repens L., R.bulbosus L.;R.acris. III. A mathematical model incorporating multiple modes of reproduction. J.Ecol. $62,3: 921-937$.

TURKINGTON, R., CAHN, M.A., VARDY, A. y HARPER, J.L. 1979 . The growth, distribution and neighbour relationships of Trifolium repens in permanent pasture. III. The establishment and growth of Trifolium repens in natural and pasture sites. J.ECoZ.67, I' 231-243.

USHER, M.B. 1972 . Developments in the Leslie Matrix Model. En J. N.R. Jeffers (ed.) Mathematical Models in Ecology. Blackwell's Scientific Publications.

WATKINSON, A.R. y HARPER, J.L. 1978 . The demography of a sand du ne annual : Vulpia fasciculata $I$. The natural regulation of populations . J.Ecol. 66,1:15-35.

WERNER, P, y CASWELL, H. 1977 . Population growth rates and age versus stage-distribution models for teasel (Dipsacus sylves tris huds). Ecology 58,5:1103-1112. 
WHITTAKER, R.H. y WOODWELL, G.M. 1969 . Structure production and diversity of the oak-pine forest at Brookhaven, New York . J. Ecol. 57, 1: 155-175.

WHITTAKER, R.H. y GOODMAN, D. 1979 . Classifying species according to they demographic strategy. I. Population fluctuations and environmental heterogenity . Amer. Naturalist 113,2:185-200.

ZIMMERMAN, M.H. y BROWN, C.L. 1971 . Trees structure and function. Springer-Verlag. Berlin. 